\title{
Práticas de Supervisão Pedagógica: Análise dos Relatórios de Avaliação Externa de Escolas
}

\section{Monitoring Classroom Practices: \\ An analysis of the Reports of the External Evaluation of Schools}

\author{
Margarida Amaral*, Graça Bidarra**, Piedade Vaz-Rebelo**, Carlos Barreira** \\ *Agrupamento de Escolas de Seia, **Universidade de Coimbra
}

\begin{abstract}
Resumo
Pretendendo identificar práticas de supervisão em escolas que se destacaram no primeiro e segundo ciclos avaliativos do programa de Avaliação Externa de Escolas no domínio Prestação de serviço educativo, procedeu-se à análise de conteúdo dos relatórios de seis escolas do primeiro ciclo avaliativo com a classificação de Muito Bom tanto no domínio referido como no fator Acompanhamento da prática letiva e de duas no segundo ciclo avaliativo que mantiveram os requisitos estabelecidos. A análise efetuada revela que as escolas desenvolveram práticas indiretas de supervisão pedagógica, embora, no segundo ciclo, a partilha de materiais, o trabalho colaborativo entre pares e a ação supervisiva das lideranças intermédias também sejam referidas.

Palavras-chave: avaliação de escolas, supervisão pedagógica, prática letiva, trabalho colaborativo.
\end{abstract}

\begin{abstract}
Wishing to identify pedagogical supervision practices in schools that stood out in the first and second cycles of evaluation program of EES in Provision of Educational Service, an analysis was made on the contents of reports of six schools in the first evaluation cycle with MB both in the area referred to as the factor Monitoring Classroom Practices and two in the second evaluation cycle that kept the requirements established. This analysis reveals that the schools researched developed indirect pedagogical supervision practices, although, in the second cycle, the sharing of materials, collaborative work between pairs and the action of supervision from intermediate leaders, are also referred.

Keywords: school evaluation, pedagogical supervision, classroom practice, collaborative work.
\end{abstract}

\section{Introdução}

Os estudos sobre a eficácia e qualidade educativa têm vindo a procurar combinar duas vias de investigação que permaneciam relativamente separadas - a eficácia e qualidade da escola e a eficácia e qualidade do trabalho do professor. Embora com objetivos e metodologias de investigação diferentes, estes dois tipos de investigação que decorrem de campos científicos igualmente diferentes (Sociologia/Administração Educativa e Psicologia Educacional) tendem a aproximar-se nos estudos mais recentes. É neste contexto, que procuramos estudar o contributo da supervisão pedagógica para a eficácia das escolas, a importância que lhe é atribuída, as modalidades de que se reveste, tendo como ponto de partida a análise dos relatórios de avaliação externa das escolas da zona centro de Portugal, relativos aos primeiro e segundo ciclos avaliativos (2006-2011 e 2012-2014, respetivamente), com particular ênfase no domínio da Prestação do serviço educativo, de acordo com o quadro de referência adotado.

No âmbito da investigação sobre as escolas e a sua qualidade, as atividades de sala de aula assumem um papel relevante na explicação dos resultados académicos dos alunos (Lima, 2008; Scheerens, 2004), justificando o interesse em torno dos modelos teóricos e das estratégias para monitorizar essas práticas, desenvolvidos, tanto a nível internacional (Duffy, 1998; Glickman, Gordon \& Ross-Gordon, 2013) como, a nível nacional, com os trabalhos realizados em supervisão pedagógica (Alarcão \& Tavares, 2007; Formosinho, 2002; Vieira \& Moreira, 2011).

A nível nacional, os relatórios do Conselho Nacional de Educação (2002; 2005; 2008) sobre a avaliação externa das escolas também afirmam que os fatores considerados mais relevantes para a melhoria das organizações escolares focam os processos que ocorrem na sala de aula, destacando a importância do papel do professor e da supervisão pedagógica como uma ação unificadora capaz de gerar práticas fundamentais para melhorar a eficácia do ensino e das escolas.

Identificar as vantagens da supervisão pedagógica numa perspetiva de regulação associada às funções dos coordenadores dos departamentos curriculares e / ou dos subcoordenadores de grupo pedagógico, é, um dos nossos objetivos.

As dimensões da supervisão pedagógica englobam a indicação de uma direção para a prática de ensino através de um acompanhamento contínuo. De acordo com Isabel 
Alarcão (2009, p. 121), referindo-se aos professores, considera que todos somos supervisores no sentido de que todos temos o dever de inter-ajudar e contribuiir para uma escola melhor, defendendo "a supervisão como um processo de criação de contextos de aprendizagem" (Alarcão, 2009, p.125), sem ser necessário definir os supervisores institucionais. Esta ideia reforça a importância do conhecimento das práticas de supervisão pedagógica no campo da Prestação do serviço educativo.

\section{Metodologia}

A metodologia desenvolvida envolveu um estudo empírico documental, por meio da análise dos relatórios de avaliação externa das escolas para o primeiro e segundo ciclos de avaliação. Os anos letivos de 2007/08, 2008/09 e 2009/10 foram tidos em conta em relação ao primeiro ciclo avaliativo, tendo sido analisados 167 relatórios. Relativamente ao segundo ciclo avaliativo foram analisados os relatórios da avaliação externa de escolas dos anos letivos 2011/12, 2012/13 e 2013/14, num total de 100 .

Tendo como objetivo identificar práticas de supervisão em escolas que se destacaram no primeiro e segundo ciclos avaliativos do programa de Avaliação Externa de Escolas no domínio Prestação do serviço educativo, analisaram-se as escolas que, no primeiro ciclo avaliativo, tinham sido avaliadas com Muito Bom simultaneamente no domínio Prestação do serviço educativo e no fator Acompanhamento da prática letiva, num total de seis escolas. Procurou-se verificar a sua classificação no mesmo domínio, no segundo ciclo avaliativo, tendo-se constatado que das três escolas à data avaliadas, apenas duas mantiveram a classificação obtida. Procedeu-se então à análise de conteúdo dos relatórios de avaliação externa destas escolas, no primeiro e no segundo ciclo avaliativos, considerando como corpus o domínio da Prestação do serviço educativo. No que diz respeito ao primeiro ciclo de avaliação, analisou-se o fator Acompanhamento da prática letiva, tendo-se estendido a análise a todo o domínio da Prestação do serviço educativo, no segundo ciclo, pois em todos os seus campos de análise existem referentes relacionados com o Acompanhamento da prática letiva.

\section{Resultados}

A frequência das classificações no domínio Prestação de Serviço educativo, no primeiro ciclo avaliativo, por ano letivo, é descrita na Figura 1.

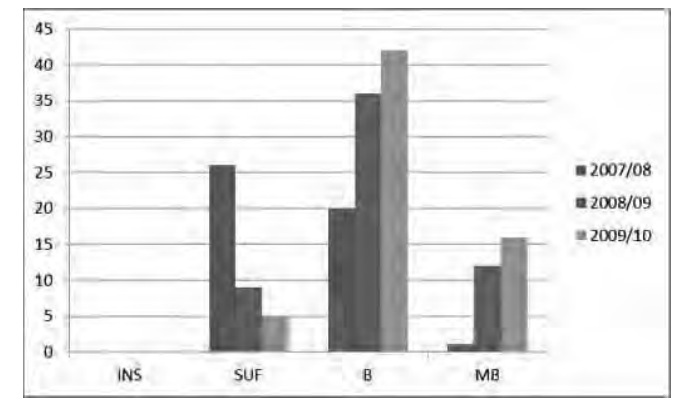

Figura 1. Frequência das classificações no domínio Prestação de serviço educativo, $1^{\circ}$ ciclo
Para o segundo ciclo avaliativo a descrição das frequências das classificações no domínio Prestação do serviço educativo é feita na figura 2.

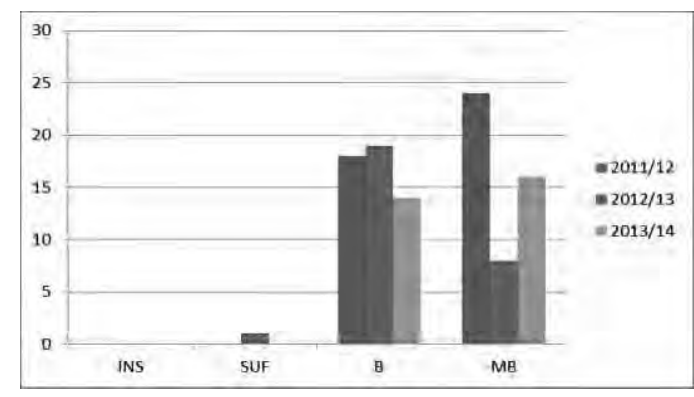

Figura 2. Frequência das classificações no domínio Prestação de serviço educativo, $2^{\circ}$ ciclo

Ao longo dos anos a variação da frequência apresenta-se na figura 3 .

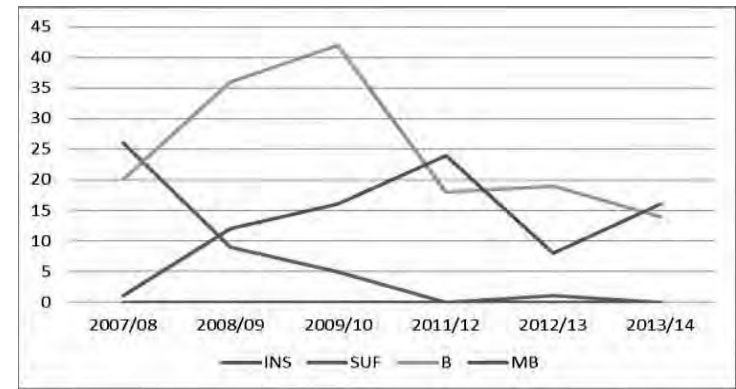

Figura 3. Variação das classificações no domínio Prestação de serviço educativo ao longo dos anos letivos

Para a análise de conteúdo dos relatórios de avaliação externa das escolas no primeiro e segundo ciclos avaliativos tomou-se como corpus o domínio da Prestação do serviço educativo e as subcategorias de acordo com o referencial adotado para o $2^{\circ}$ ciclo, o que obrigou a algumas adaptações, no que se refere aos dados do primeiro ciclo.

As subcategorias consideradas foram então: Planeamento e articulação; Práticas de ensino $e$ Monitorização e Avaliação do ensino e das aprendizagens. Os indicadores por subcategoria são apresentados no Quadro 1.

$\mathrm{O}$ estudo realizado com base na análise dos relatórios das seis escolas avaliadas com MB no $1^{\circ}$ ciclo de avaliação mostrou que estas desenvolviam, predominantemente, práticas indiretas de supervisão pedagógica, especialmente ao nível da avaliação, estendendo-se às práticas de ensino e ao planeamento. $\mathrm{O}$ número de unidades de registro por subcategoria é apresentado na Figura 4.

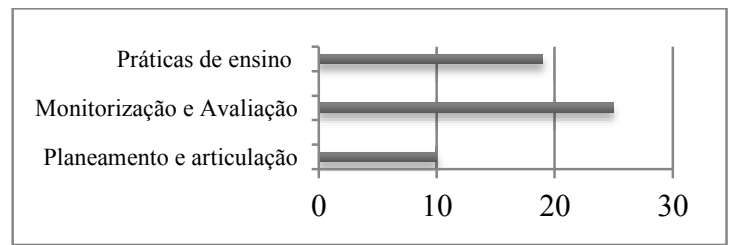

Figura 4.Unidades de registo por subcategoría para o primeiro ciclo avaliativo 
Quadro 1

Subcategorias e indicadores do domínio Prestação de serviço educativo

\begin{tabular}{cc}
\hline Subcategorias & Indicadores \\
\hline Planeamento e articulação & Gestão articulada do currículo \\
& Contextualização do currículo e abertura ao meio \\
& Utilização da informação sobre o percurso escolar dos alunos \\
Coerência entre ensino e avaliação \\
Trabalho cooperativo entre docentes \\
\hline Práticas de ensino & Adequação das atividades educativas e do ensino às capacidades e aos ritmos de \\
aprendizagem das crianças e dos alunos
\end{tabular}

\section{Quadro 2}

Frequências das unidades de registo associadas aos indicadores no primeiro e segundo ciclos

(Subcategorias.Planeamento e articulação $\square$; Práticas de ensino $\square$ Monitorização e avaliação do ensino e das aprendizagen $\square$ )

\begin{tabular}{|c|c|c|c|}
\hline \multirow{2}{*}{ Indicadores } & \multicolumn{3}{|c|}{ Frequência das Unidades de Registo } \\
\hline & $1^{o}$ cicclo & $2^{o}$ ciclo & Total \\
\hline Gestão articulada do currículo & - & 1 & 1 \\
\hline Contextualização do currículo e abertura ao meio & - & 1 & 1 \\
\hline Utilização da informação sobre o percurso escolar dos alunos & 1 & 1 & 2 \\
\hline Coerência entre ensino e avaliação & 2 & 2 & 4 \\
\hline Trabalho cooperativo entre docentes & 1 & 3 & 4 \\
\hline $\begin{array}{l}\text { Adequação das atividades educativas e do ensino às capacidades e aos ritmos de } \\
\text { aprendizagem das crianças e dos alunos }\end{array}$ & - & 1 & 1 \\
\hline $\begin{array}{c}\text { Adequação das respostas educativas às crianças e aos alunos com necessidades } \\
\text { educativas especiais }\end{array}$ & 1 & 1 & 2 \\
\hline Exigência e incentivo à melhoria de desempenhos & 1 & 4 & 5 \\
\hline Metodologias ativas e experimentais no ensino e nas aprendizagens & 1 & 1 & 2 \\
\hline Valorização da dimensão artística & 1 & - & 1 \\
\hline Rendibilização dos recursos educativos e do tempo dedicado às aprendizagens & - & 1 & 1 \\
\hline Acompanhamento e supervisão da prática letiva & 1 & 3 & 4 \\
\hline Diversificação das formas de avaliação & 1 & 2 & 3 \\
\hline Aferição dos critérios e dos instrumentos de avaliação & 2 & 4 & 6 \\
\hline Monitorização interna do desenvolvimento do currículo & 1 & 1 & 2 \\
\hline Eficácia das medidas de promoção do sucesso escolar & 1 & 2 & 3 \\
\hline Prevenção da desistência e do abandono & 1 & 2 & 3 \\
\hline
\end{tabular}

No Quadro 2 apresentam-se as frequências das unidades de registo associadas aos indicadores e subcategorias, resultantes da análise de conteúdo dos relatórios do segundo ciclo de AEE das escolas em estudo.

É interessante constatar que em todos os relatórios se faz referência à exigência e incentivo, à melhoria de desempenhos e ainda à aferição dos critérios e dos instrumentos de avaliação, como indicadores predominantes. Pode-se constatar também, que as subcategorias Práticas de ensino e Monitorização e avaliação são as que apresentam maior número de unidades de registo. 
Centrando a atenção sobre as duas escolas que foram avaliadas com MB nos dois ciclos avaliativos, no domínio Prestação de serviço educativo chegámos aos resultados apresentados na figura 5 .

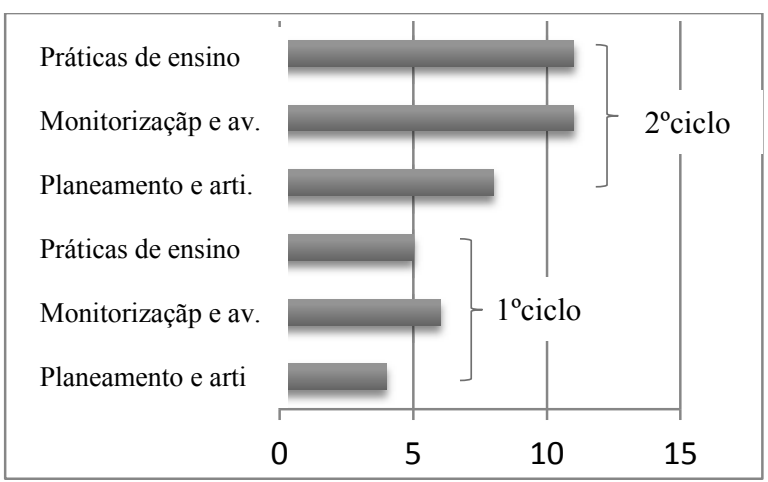

Figura 5.Unidades de registo por subcategoria relativas às escolas avaliadas nos dois ciclos

\section{Conclusão}

Os resultados obtidos a partir da análise de conteúdo dos relatórios de AEE das escolas em estudo permitem-nos observar que, embora a subcategoria Monitorização e avaliação do ensino e das aprendizagens seja a mais relevante nos primeiro e segundo ciclos de avaliação, neste último ganha igual relevância a subcategoria Práticas de ensino. Refira-se que é o indicador Acompanhamento e supervisão da prática letiva que corresponde a um aumento de unidades de registo de um ciclo para o outro, isto porque é tomada em consideração uma maior ação supervisiva por parte das lideranças intermédias (sem observação de aulas) sobre as atividades letivas, nomeadamente quanto aos seus efeitos na aprendizagem dos alunos. Para Scheerens (2004), os líderes intermédios de sucesso envolvem-se diretamente no trabalho que é feito com os alunos, avaliando sistematicamente as suas dinâmicas de sala de aula e as dos seus colegas, monitorizam os progressos e resultados dos alunos, promovendo um ambiente de trabalho propício à aprendizagem. Nesta perspetiva trata-se de uma prática supervisiva em crescendo nas nossas escolas. Para que seja possível o desenvolvimento de tal prática, são necessários docentes detentores de atitudes de colaboração e partilha, o que está de acordo com os resultados encontrados. Pois, de facto, também é notório o aumento de unidades de registo referente ao indicador Trabalho cooperativo entre docentes, sendo a partilha de materiais e o trabalho colaborativo entre pares referidos mais frequentemente no segundo ciclo avaliativo.

\section{Referências}

Alarcão, I. (1999). Um olhar reflexivo sobre a supervisão. In Supervisão na formação - Contributos Inovadores. Actas do $10^{\circ}$ Congresso Nacional de Supervisão.

Alarcão, I. (2009). Formação e supervisão de professores: uma nova abrangência. Sísifo Revista de Ciência da Educação, nº 8, (pp 119-128).
Alarcão, I., \& Tavares, J.(2003). Supervisão da prática pedagógica: Uma perspectiva de desenvolvimento e aprendizagem. Lisboa: Livraria Almedina.

Barreira, C. F, Bidarra, M. G., \& Vaz-Rebelo, P. (2011). Avaliação externa de escolas: Do quadro de referência aos resultados e tendências de um processo em curso.

Revista Portuguesa de Pedagogia, Extra-Série, 81 - 94.

Duffy, F. M. (1998). The ideology of supervision. In G.

Firth \& E. Pajak (Eds.) Handbook of Research on

School Supervision (pp. 181-199). New York:

Macmillan.

Formosinho, J. (Org.) 2002. A supervisão na formação de professores II; Da organização à pessoa. Porto: Porto Editora.

Glickman, C., Gordon, S.P., \& J. M Ross-Gordon (2013). Supervision and instructional Leadership: A developmental approach. Boston: Pearson Education. Inspeção Geral da Educação (2006-2011). Avaliação Externa das Escolas: Aaliar para a melhoria e confiança. Ministério da Educação - IGE.

Lima, J. A. ( 2008). Em busca da boa escola: Instituições eficazes e sucesso educativo. Vila Nova de Gaia:

Fundação Manuel Leão.

Scheerens, J. (2004). Melhorar a eficácia das escolas. Porto: Edições ASA

Simões, H., Nascimento, M. A., \& Vaz-Rebelo, P. (2012). Teachers' supervision in Portugal: Concepts and practices. In A. Swennen et al. (Org.). Book of Abstracts. IV ATEE Winter Conference. Professional development of teacher educators: Bringing together policy, practice and research. University of Coimbra. p.119.

Vieira, F, \& Moreira, M. A. (2011). Supervisão e avaliação do desempenho docente: Para uma abordagem de orientação transformadora. Lisboa: Ministério da Educação. 